\title{
Membranas cerámicas y su utilidad en procesos de separación
}

\author{
A. HERNÁNDEZ, P. PRÁDANOS, J. I. CALVO Y L. PALACIO \\ Dpto. Termodinámica y Física Aplicada, Facultad de Ciencias, Universidad de Valladolid, 47071 Valladolid, España
}

\begin{abstract}
Entre los procesos industriales de separación, los que se basan en membranas se consideran como un nuevo y prometedor campo en desarrollo. Entre éstos, los basados en el uso de membranas cerámicas por sus características de resistencia mecánica, térmica y química y cada vez mayor selectividad han adquirido una gran relevancia. En este trabajo se comenzará haciendo una breve descripción de los métodos de síntesis de membranas inorgánicas y en particular cerámicas. Se exponen tanto los métodos tradicionales como los de sol-gel por vías polimérica y coloidal. Para caracterizar estas membranas se deben obtener sus distribuciones de tamaño de poro y propiedades superficiales tanto morfológicas como relacionadas con la hidrofílicidad y sus propiedades eléctricas. Algunas técnicas de investigación de estas propiedades estructurales y superficiales se exponen también resumidamente. Las membranas cerámicas pueden usarse en las mismas aplicaciones que las membranas poliméricas, con la ventaja de sus mejores prestaciones y durabilidad.
\end{abstract}

Palabras Clave: Materiales porosos, Membranas cerámicas, Procesos de Separación, Ultrafiltración, Microfiltración, Nanofiltración.

Ceramic membranes and their use in separation processes.

Among the separation processes in the industry, those using membranes are usually considered as forming a new and promising field. From them, those which use ceramic membranes, which can be manufactured more selective now than some time ago, are especially relevant due to their high mechanic, thermal and chemical resistances.Here we will start by a summary description of the methods of synthesis of inorganic membranes and particularly ceramic ones. Traditional methods are presented as well as both the polymeric and the coloidal sol-gel techniques. In order to characterize them, pore size distributions and surface properties, including morphology, hydrophillicity and electrical properties should be investigated. Some techniques to study these structural and surface properties are summarily presented. The ceramic membranes can substitute polymeric ones in most of their typical applications with profit due to their better performances and durability.

Key words: Porous materials, Ceramic membranes, Separation processes, Ultrafiltration, Microfiltration, Nanofiltration.

\section{INTRODUCCIÓN}

Aún ahora, se sigue considerando a los procesos de membrana como un campo nuevo y prometedor en el área de los procesos industriales de separación. Sin embargo, lo cierto es que teniendo en cuenta la cantidad de procesos industriales en que la separación por medio de membranas es ya el método estándar, se debe concluir que la ciencia y la tecnología de membranas están plenamente consolidadas.

El factor clave en la utilización de una membrana es la selectividad, ya que el carácter de ésta define el proceso de membrana y la aplicación para la que puede ser adecuado. Los procesos de membrana más utilizados en la actualidad se muestran en la Figura 1, comparados con otros procesos más tradicionales de separación (1). Está claro que para ser utilizadas en tan amplio rango de aplicaciones y procesos, las membranas deben ser muy diferentes en estructura, propiedades y fabricación. Una primera clasificación se puede acometer basándose en aspectos estructurales (relacionados lógicamente con los funcionales) (2). Una clasificación de ese tipo se muestra en la Figura 2.

En cuanto a lo que se refiere al material de la membrana (o materiales en el caso de las membranas compuestas), muchos de ellos han sido propuestos y usados en los aproximadamen- te 40 años de investigación y aplicación de los procesos de membrana, (3). El uso de materiales inorgánicos es relativamente nuevo pero está expandiéndose rápidamente con un amplio rango de aplicaciones potenciales. Mucho del interés que existe en las membranas inorgánicas es debido a las ventajas que estos materiales ofrecen sobre los poliméricos, (4). Entre ellas se pueden mencionar: una alta resistencia a muchas especies químicas incluyendo productos corrosivos; una gran tolerancia a un amplio rango de $\mathrm{pH}$ y temperaturas así como una alta resistencia mecánica a la presión. Especialmente importante es la posibilidad de esterilización repetida, lo cual hace a las membranas inorgánicas muy interesantes cuando se trata con especies como las farmacéuticas, biotecnológicas o alimentarias. Estas ventajas, evidentes, deben considerarse junto con algunas desventajas como son su mayor costo inicial y su fragilidad. En cualquier caso su mayor estabilidad hace posible una limpieza eficaz y, por tanto, una mayor vida operativa. Algunas de estas ventajas y desventajas se muestran en la Tabla I.

En este trabajo pretendemos exponer muy brevemente algunos de los aspectos más relevantes concernientes a las membranas inorgánicas, particularmente cerámicas. Esta exposi- 
ción comenzará estudiando los diferentes métodos de fabricación. Seguiremos exponiendo algunos métodos de caracterización estructural y funcional, para finalizar con una breve relación de las principales aplicaciones industriales de tales membranas.

\section{MATERIALES POROSOS Y MEMBRANAS}

\subsection{Síntesis}

Las membranas cerámicas se pueden sintetizar por varios métodos, además pueden formar parte de membranas compuestas inorgánicas, cuyos substratos no sean cerámicos. Por lo que un adecuado conocimiento de cuales son los principales métodos de fabricación de membranas inorgánicas en general resulta imprescindible.

Las membranas inorgánicas fueron inicialmente utilizadas (aun hoy ése es uno de los más relevantes campos de aplicación) en el enriquecimiento en $U^{235}$ del mineral de uranio mediante difusión gaseosa del $\mathrm{UF}_{6}$ en membranas porosas. Esto ocurría en las décadas de los 40 y 50. Aunque una exposición de su desarrollo histórico, (5), estaría fuera de lugar, digamos que, en los años 80 y 90, el paulatino abandono de la energía nuclear hizo redirigir los esfuerzos ya hechos a otros procesos y aplicaciones de las membranas. En la actualidad existen un gran número de membranas inorgánicas (muchas de ellas cerámicas, al menos en parte) y empresas fabricantes, (6).

TABLA I.

VENTAJAS DESVENTAJAS DE LAS MEMBRANAS INORGÁNICAS.

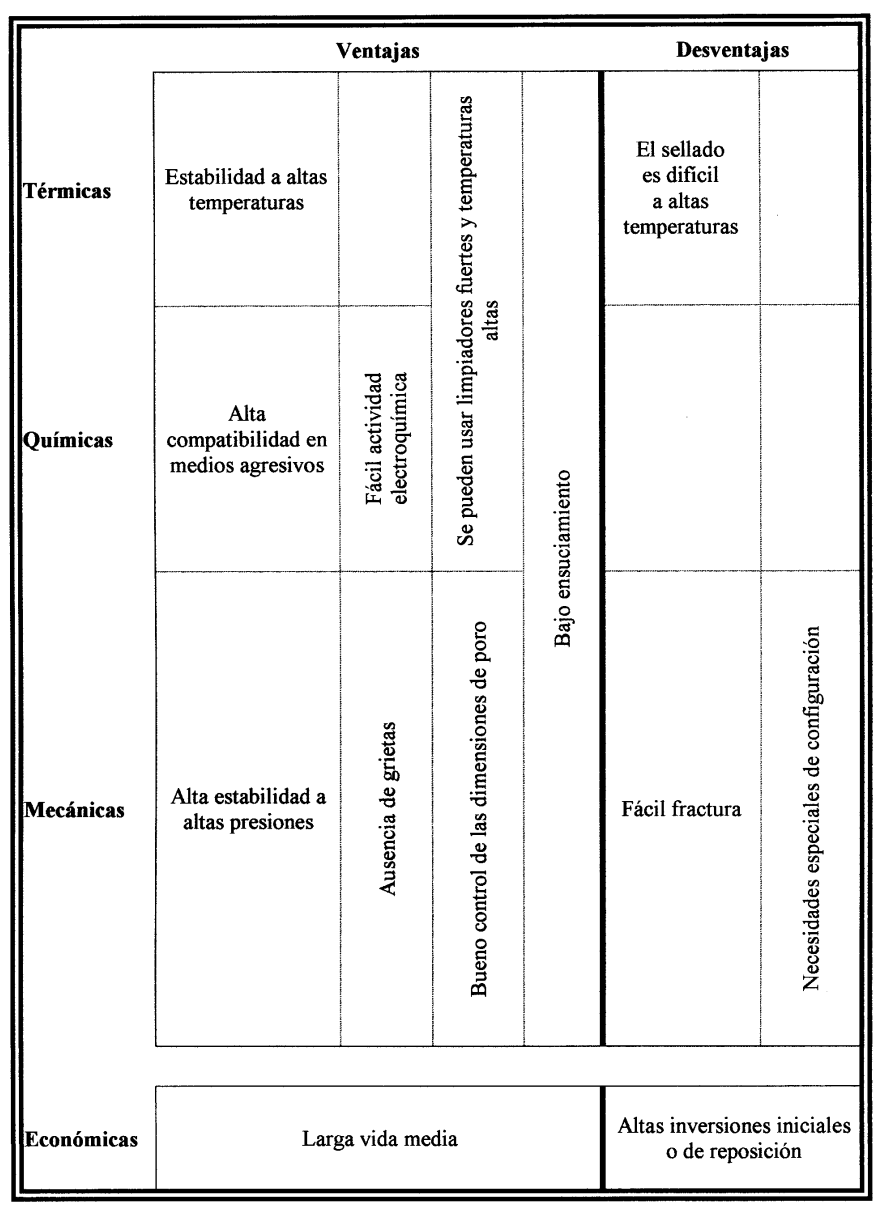

\subsubsection{MEMBRANAS NO CERÁMICAS}

Los óxidos o ceratos estables de zirconio y bismuto en capas sólidas muy finas son permeables a las formas iónicas de hidrógeno y oxígeno, por lo cual han sido usadas especialmente en reactores de membrana. A fin de obtener permeabilidades significativas, estas membranas deben ser muy finas, por lo cual se necesita depositarlas sobre un soporte poroso apropiado. Otras membranas densas se obtienen rellenando una membrana porosa con un líquido que permanece inmóvil controlando la permselectividad, (5). También se pueden obtener membranas porosas en el rango nanométrico mediante pirólisis controlada de: polímeros térmicamente tratados (para dar membranas de carbono); o gomas de silicona, para obtener filtros de silicio.

Se pueden obtener membranas de poros muy regulares en forma y tamaño por el método de revelado de trazas nucleares. Una capa delgada $(\sim 10 \mu \mathrm{m})$ y sólida es bombardeada por partículas muy energéticas (fragmentos de fisión o iones acelerados), de forma que dichas partículas producen daños en el material que pueden ser posteriormente revelados de forma química. Este método se utiliza por ejemplo con mica, así como con materiales metálicos.

\subsubsection{MEMBRANAS CERÁMICAS}

Las membranas inorgánicas más desarrolladas y utilizadas son las cerámicas. Una cerámica se puede definir como un material policristalino consolidado basado en compuestos de elementos de los grupos III a VI. La estructura, la porosidad y el tamaño de poro están directamente determinados por el método utilizado para transformarlas. Así poros más anchos se pueden obtener por los métodos ceramistas tradicionales mientras que los poros más estrechos necesitan técnicas más modernas como el método sol-gel. Un breve resumen de dichas técnicas se muestra en la Figura 3, (7).

Fuera de ese esquema, se hallan algunos procesos muy específicos, como la oxidación anódica de aluminio. Este proceso, en un determinado electrólito ácido, da lugar a una estructura de celdas hexagonales de aluminio, conteniendo en su interior

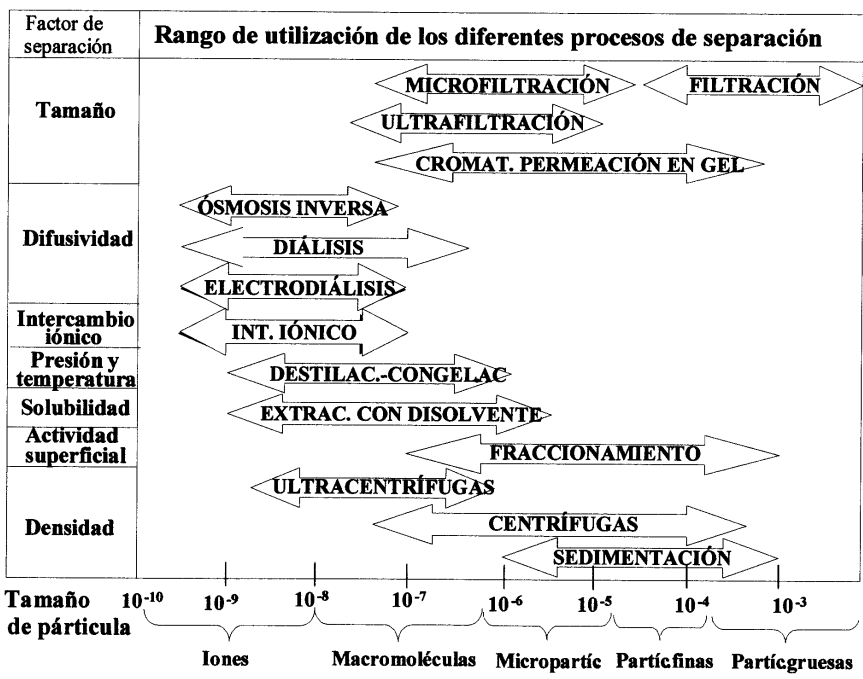

Fig. 1.- Los procesos de membrana más utilizados en la actualidad comparados con otros procesos más tradicionales de separación 
poros cilíndricos. El tamaño de celda y poro se controlan mediante el voltaje de anodizado, mientras que el espesor de la película lo determina la densidad de corriente y el tiempo de anodizado. Las capas porosas obtenidas se desligan del substrato de aluminio mediante reducción controlada del voltaje, lo cual da lugar a una capa fina formada por poros más pequeños (4).

\subsubsection{Técnicas cerámicas tradicionales}

Desde el punto de vista de fabricación de membranas, estas técnicas se utilizan mayormente para realizar soportes macroporosos que pueden ser usados como membranas de microfiltración. Sin embargo, se obtienen resultados pobres, con distribuciones de tamaño de poro demasiado amplias así como la presencia de numerosos defectos que conducen a la rotura o formación de agujeros. Para mejorar su calidad generalmente se deposita una capa selectiva por el método de sol-gel.

Las técnicas clásicas de hacer cerámicas consisten en la deposición o moldeo de una suspensión en polvo. Así los principales pasos son: preparación del polvo, moldeado o deposición, secado y sinterizado (vease la Figura 3). Los factores más importantes en tanto que determinan el producto final son el tamaño de grano y los aditivos, dado que ellos controlan la microestructura resultante. Cuando se parte de un óxido puro, son necesarios varios aditivos (frecuentemente inorgánicos), los cuales deben ser eliminados por tratamiento térmico (7).

\subsubsection{Técnica Sol-Gel}

Esta técnica permite obtener materiales cerámicos a partir de una disolución. Las partículas de partida son más pequeñas (unos pocos nanómetros) que con el uso de las técnicas tradicionales, debido a que son obtenidas por hidrólisis de compuestos órgano-metálicos u oxidación de sales. Es por ello que las cerámicas obtenidas son más densas, con mayor resistencia a la compresión. Asimismo las imperfecciones son más pequeñas y menos frecuentes que cuando se usan los otros métodos (de hecho presentan escasas grietas e inferiores a una micra en tamaño).

Después de la hidrólisis controlada, todas las partículas tienen tamaños muy similares, (2-3 nm usualmente), estabilizadas (mediante la adición de productos químicos cuidadosamente escogidos) por su carga o la presencia de un polímero

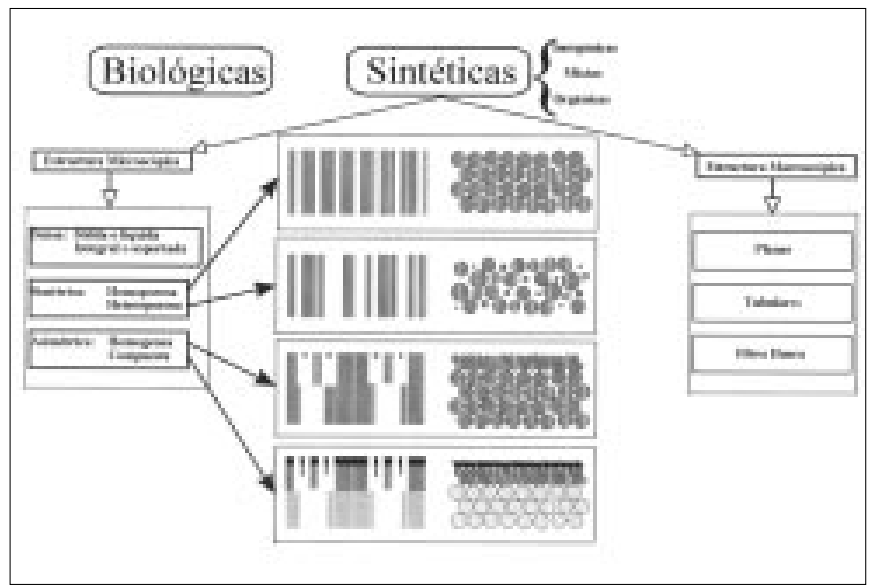

Fig. 2.- Clasificación de las membranas basada en aspectos estructurales. orgánico en la llamada fase sol. A concentraciones apropiadas, estas partículas se unen para formar un sólido homogéneo tridimensional que se separa del líquido en la fase gel. Finalmente, después del secado y sinterizado se obtiene la cerámica definitiva (5).

La técnica sol-gel puede seguir dos caminos, dependiendo del material de partida (vease la figura. 3):

1. Ruta coloidal, que comienza por una sal metálica, dando tras hidrólisis una suspensión coloidal de óxidos e hidróxidos metálicos, con formación de grupos crecientes que debido a colisiones condensan para formar un gel, (4). Las características del gel cerámico así obtenido dependen del potencial zeta de las partículas del sol, que es controlado cambiando $\mathrm{pH}$ y concentración de sal o fuerza iónica.

2. La ruta del gel polimérico comienza por compuestos órgano-metálicos, normalmente alkóxidos unidos al metal por su oxígeno. Aquí el alkóxido se hidroliza y condensa para formar una solución viscosa de polímeros órgano-metálicos. Por un adecuado control los clusters poliméricos crecen en la fase sol hasta la gelificación.

En ambas técnicas son necesarios aditivos específicos de la ruta, pero también otros que son usados para mejorar la reología del sol permitiendo un moldeo más sencillo. Normalmente, se usa la deposición en placa sobre un soporte apropiado, seguido de gelificación in situ, secado y sinterizado. En ambos casos los compuestos orgánicos se queman durante el sinterizado.

\subsection{Caracterización}

La caracterización estructural de una membrana supone, fundamentalmente, la determinación experimental de los siguientes parámetros: distribución estadística de tamaños de poro; tanto por ciento de volumen poroso o porosidad y rugosidad superficial. En cuanto a la caracterización funcional de las membranas lo que se estudia es la permeabilidad, los coe-

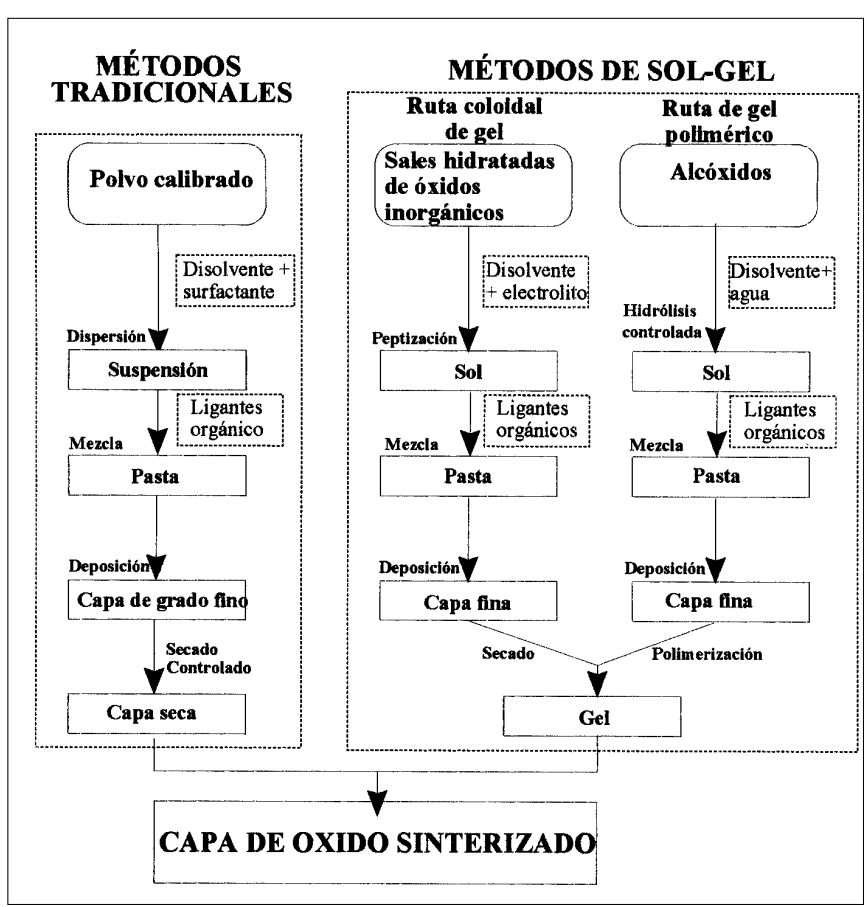

Fig. 3.- Esquema de las técnicas de fabricación de membranas cerámicas. 
ficientes de retención y factores de separación, los coeficientes de difusión efectiva, las características de adsorción, así como diversas pruebas de compatibilidad química, mecánica y física. Para determinar todos estos parámetros que se han enumerando, se dispone de una serie de técnicas experimentales de caracterización más o menos complejas, que nos permitan evaluar las diversas propiedades de las membranas, (2). A continuación vamos exponer de forma muy resumida los métodos más importantes que permiten obtener la distribución de tamaños de poro en los rangos que se muestran en la Figura 4.

A) Microscopía electrónica: Estas técnicas permiten obtener imágenes tanto de la superficie como de secciones transversales de la membrana: microscopía electrónica de barrido (SEM), microscopía electrónica de transmisión (TEM), microscopía electrónica por efecto de campo (FESEM), etc. Normalmente después se hace un análisis computerizado de la imagen para obtener los distintos parámetros como la distribución de tamaño de poros, (8-10). Sendas imágenes SEM y FESEM de una membrana de (-alumina obtenida por deposición anódica se muestran en la Figura 5. En la Figura 6, por otra parte, se muestran las correspondientes distribuciones de tamaños de poro, obtenidas mediante análisis computerizado de imagen.

B) Microscopía de fuerza atómica: Esta técnica permite el estudio de materiales, tanto conductores como no conductores, llegando a una escala de nanómetros. Fue desarrollada por Binning et al., (11), y su principal ventaja, frente a la microscopía electrónica, es que no es necesaria la preparación previa de la muestra. Aunque es una técnica relativamente nueva, su aplicación al campo de las membranas tanto biológicas como sintéticas, se está extendiendo a gran velocidad, (12-14). En la Figura 7 se observan las distribuciones de tamaños de poro obtenidas mediante análisis computerizado de imágenes AFM de la misma membrana referida en las Figuras 5 y 6 . Sendas imágenes AFM de ambas caras de una membrana asimétrica con capa activa de $\mathrm{ZrO}_{2}$ y soporte de alumina se muestran en la Figura 8.

C) Métodos basados en el punto de burbuja: El método, introducido por Bechhold et al., (15), está basado en la medida de la presión necesaria para hacer fluir a través de una mem-

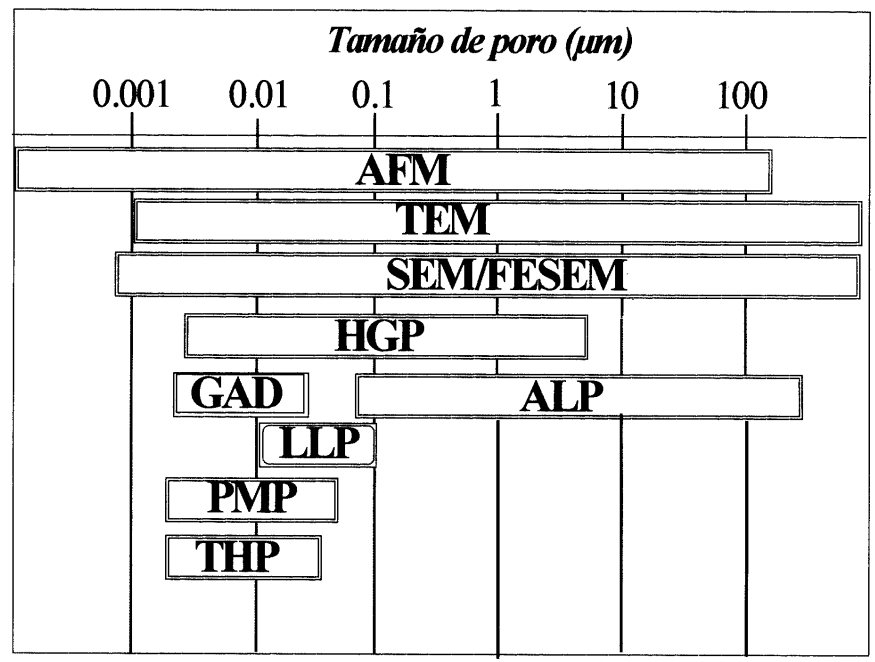

Fig. 4.- Rangos de tamaños de poro detectables mediante diversos métodos de caracterización estructural. brana el líquido en que se hallaba empapada. El punto de burbuja y métodos relacionados (tanto la técnica de desplazamiento líquido-líquido como líquido-aire) se usan muy a menudo para hacer una estimación del tamaño de poro medio y la distribución de tamaños de muchas membranas comerciales, mediante la simple determinación del punto de burbuja, siendo las técnicas estándar recomendadas, (16-19). No obstante, presenta algunos inconvenientes. Por una parte, un desconocimiento del ángulo de contacto del fluido con el material; además, en muchos casos se deben utilizar líquidos que no son los normales de operación de la membrana y que pueden modificar ligeramente su estructura. Como un ejemplo, en la Figura 9 se muestra la distribución de número de poros, obtenida con técnica de desplazamiento gas-líquido para una membrana de $\gamma$-alumina.

D) Porosimetría de mercurio: Este método está basado en los mismos principios que el método de punto de burbuja, pero en este caso se usa mercurio (un líquido que no moja) para llenar la membrana seca (20-21). Aparte del problema del ángulo de contacto ya comentado, esta técnica tiene la desventaja de que, para poros pequeños, las presiones de trabajo suelen ser tan grandes que pueden producir deformaciones

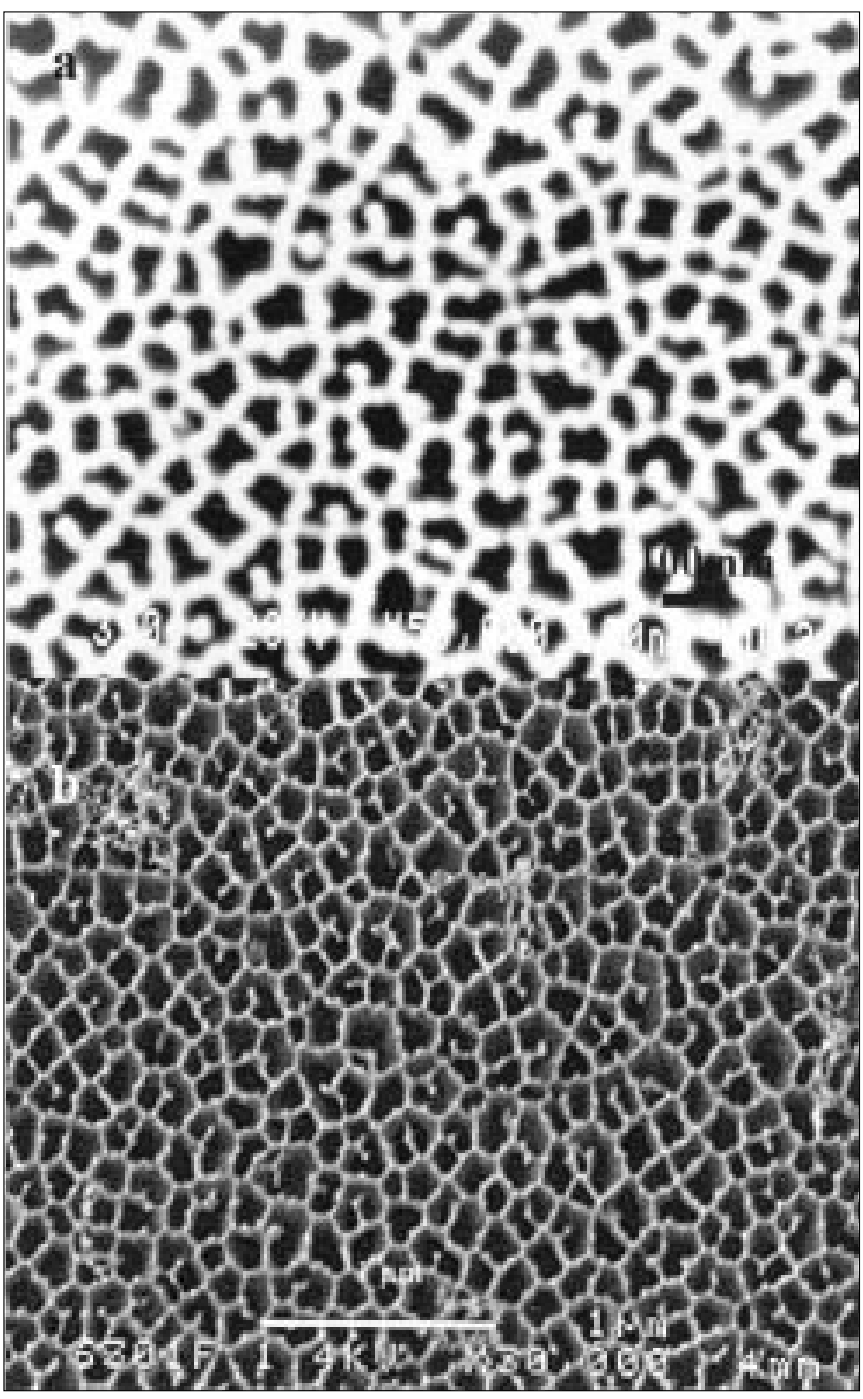

Fig. 5.- Sendas imágenes SEM y FESEM de la cara activa de una membrana ANOPORE de $\gamma$-alumina obtenida por deposición anódica. Su támaño de poro nominal es de $0,1 \mu \mathrm{m}$. 
importantes en el material. En la Figura 9 se compara la distribución de número de poros por porosimetría de $\mathrm{Hg}$ con la porometría gas-líquido. En este caso, aparece una distribución bimodal debido a la existencia de dos capas: La activa que coincide bien con la distribución de poros abiertos al flujo detectados por desplazamiento de gas-líquido y el soporte.

E) Métodos de adsorción-desorción: También es posible obtener la distribución de tamaño de poro usando dispositivos de adsorción-desorción. La técnica consiste en medir la isoterma de adsorción-desorción, y se basa en la ecuación de Kelvin, la cual relaciona la presión de vapor reducida de un líquido con una superficie curvada con la presión de vapor en el equilibrio del mismo líquido plano, (22-25). También se aplica a la isoterma de adsorción la teoría BET para obtener el área superficial específica. Este método permite determinar tamaños de poro de unos pocos angströms; aunque, por debajo de $2 \mathrm{~nm}$ no es directamente aplicable la ecuación de Kelvin. El proceso de llenado de los poros ya no es una simple condensación capilar, estando fuertemente influido por la energía de interacción con el material, con el inconveniente añadido de que el fundamento físico del proceso es poco claro. En la Figura 10 se presentan los resultados obtenidos por este método para una membrana

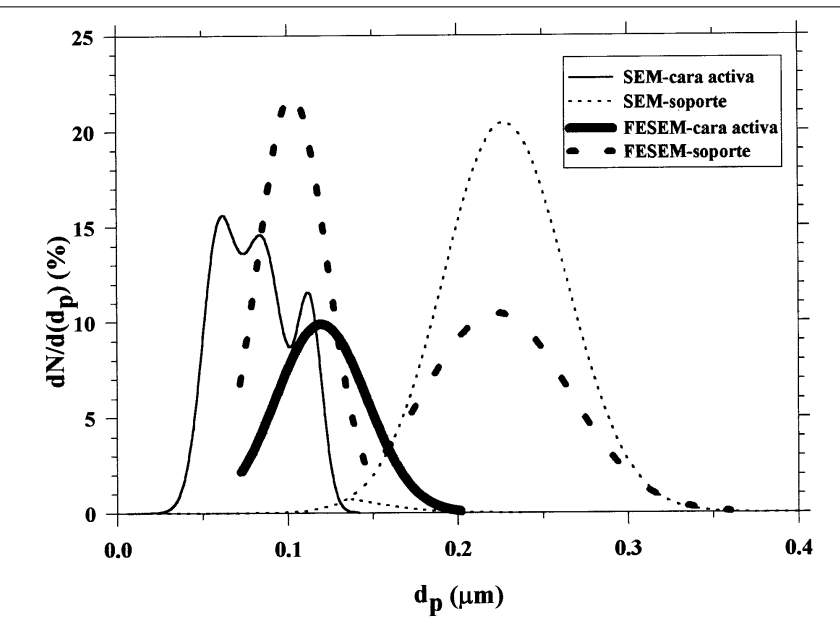

Fig. 6.- Se muestra la distribución de número de poros, obtenida mediante análisis de imágenes SEM (a) y FESEM (b) para la membrana ANOPORE de 0,1 $\mu \mathrm{m}$ de tamaño nominal de poro.

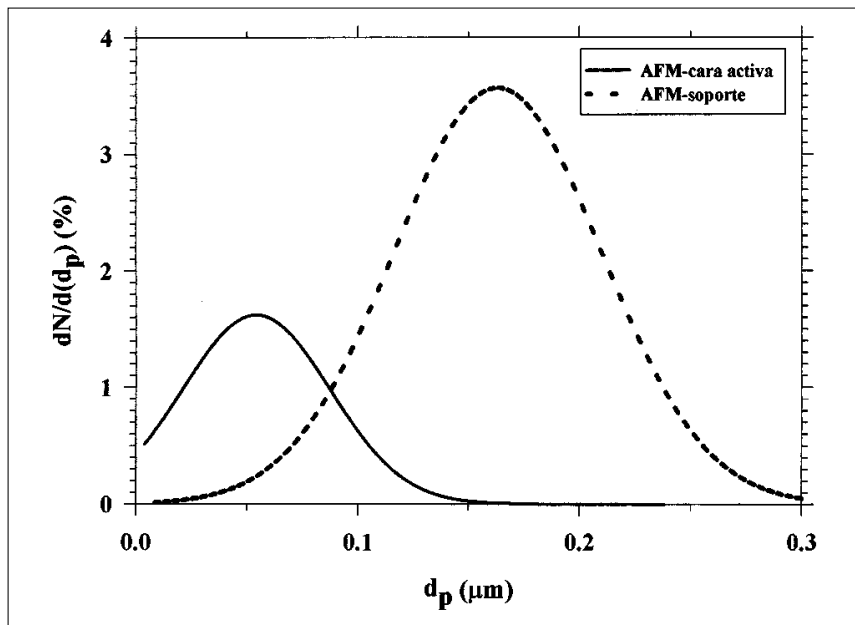

Fig. 7.- Se muestra la distribución de número de poros, obtenida mediante análisis de imágenes AFM para la membrana ANOPORE de $0,1 \mu \mathrm{m}$ de tamaño nominal de poro. de $\gamma$-alúmina, así como el ajuste de estos datos a distribuciones gausianas. Por otra parte, en la Figura 11 se comparan las distribuciones obtenidas mediante porosimetría de mercurio y adsorción-desorción de nitrógeno para la membrana TAMI mostrada en la Figura 8.

F) Permoporometría: La base de esta técnica está en el bloqueo controlado de los poros por condensación de vapor, presente en una mezcla de gases, y la medida simultánea del flujo del gas a través de los poros, $(\mathbf{2}, \mathbf{2 6})$. Así es posible obtener la distribución de tamaño de poros usando la ecuación de Kelvin. Presenta los mismos inconvenientes que el método de punto de burbuja, aunque en este caso, el gas condensable suele ser $\mathrm{O}_{2}$, o vapor de agua. El primero de ellos normalmente tiene ángulo de contacto próximo a cero con la mayoría de los materiales y no suele modificar la estructura de los materiales; el vapor de agua tiene ángulos de contacto mucho mayores, aunque conocidos. La mayor incertidumbre cuando se utiliza este método de caracterización, está en la determinación del espesor de la capa de adsorción, valor que es necesario conocer en función de la presión.

G) Termoporometría: Este método sugerido por Brun et al., (27), está basado en el hecho de que el punto de solidificación de un vapor condensado en los poros es función de la curvatura de la interfase. Usando un calorímetro diferencial de barrido (DSC) se puede observar fácilmente la temperatura de transición de fase y calcular la distribución de tamaños de poro. Es una técnica con características muy similares al método de adsorción-desorción, con el cual comparte ventajas e inconvenientes, aunque, tratándose de un cambio de fase sólido-líquido, el rango de aplicación es algo diferente.

0

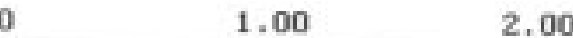

3.00

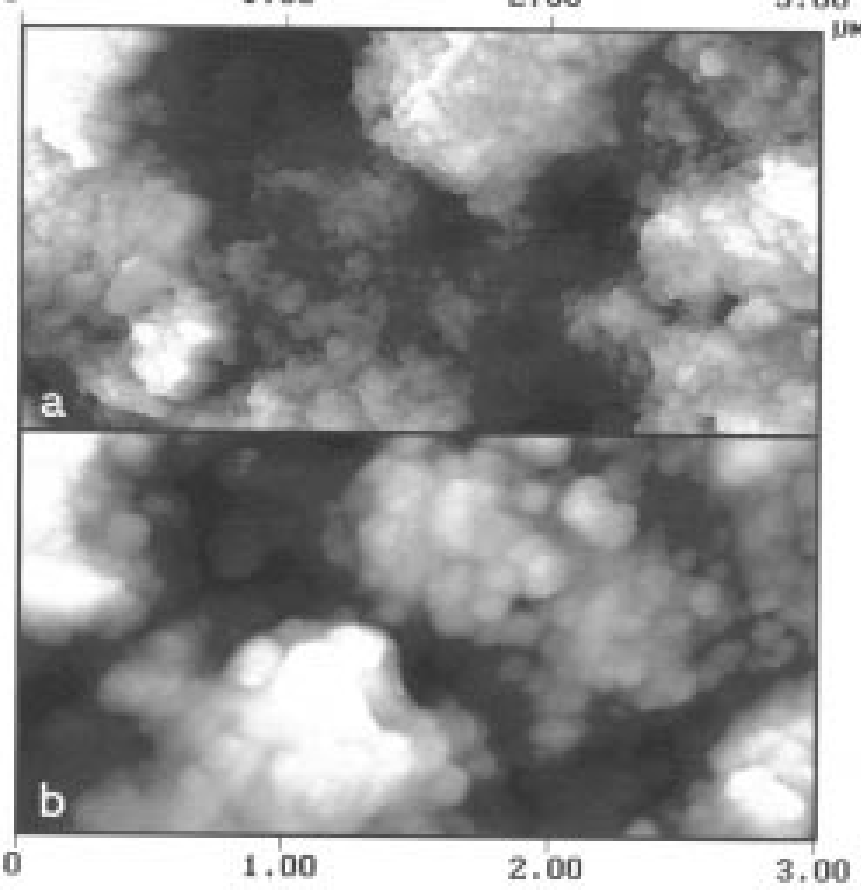

Fig. 8.- Se muestran sendas imágenes AFM de ambas caras de una membrana asimétrica de ultrafiltración con capa activa de $\mathrm{ZrO}_{2}$ (a) y soporte de alumina (b) (CÉRAM Inside), manufacturada por Tami Ind.. Se trata de una membrana de $25 \mathrm{~cm}$ de longitud y 8 canales internos, que designamos como 25TUB8. 
H) Test de retención de solutos: Se mide, para solutos de varios pesos moleculares, i.e. varios tamaños hidrodinámicos, la retención y flujo bajo unas condiciones determinadas y a partir de ahí, se calcula el tamaño de los poros de la membrana, (28-30). Éste es el método más usado y considerado como uno de los más estándar, aunque tiene el inconveniente de que es difícil de relacionar los valores de retención con los tamaños de poro. Además, está fuertemente condicionado por las condiciones experimentales exactas en que se ha realizado el proceso y por el tipo de soluto empleado. En la Figura 12 se muestra la distribución de tamaños de poro obtenida, para la membrana ANOPORE de 0,02 $\mu \mathrm{m}$ de tamaño nominal de poro, mediante experimentos de retención de proteínas de tamaños moleculares crecientes.

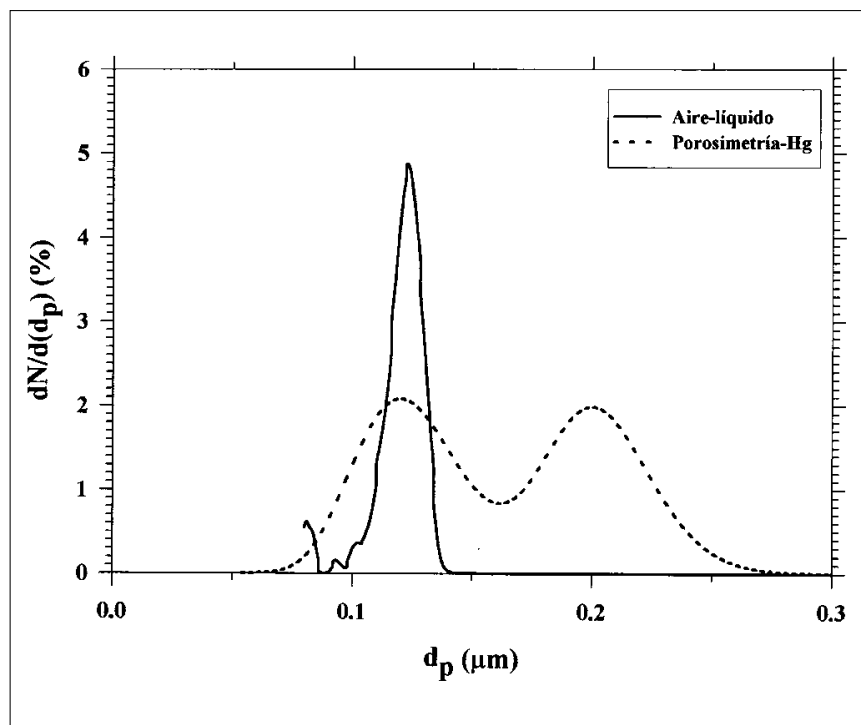

Fig. 9.- Se muestra la distribución de número de poros, obtenida con las técnicas de desplazamiento gas-líquido y porosimetría de mercurio, para la membrana ANOPORE de 0,1 $\mu \mathrm{m}$ de tamaño nominal de poro.

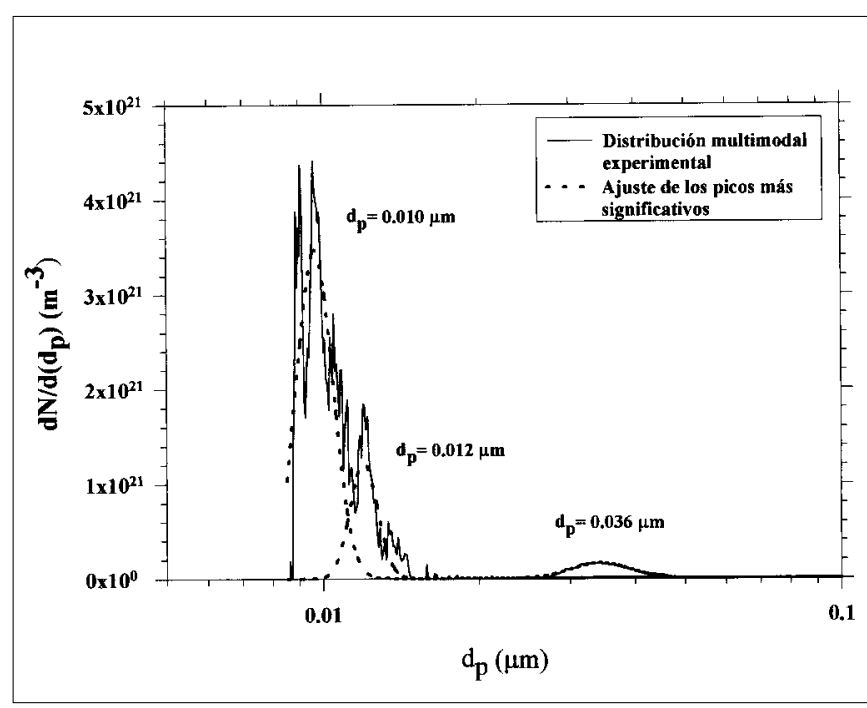

Fig. 10.- Se muestra la distribución de número de poros, obtenida mediante adsorción-desorción de nitrógeno, para una membrana ANOPORE de 0,02 um de tamaño nominal de poro.
I) Métodos espectroscópicos: Hay otras técnicas que también se pueden usar para estudiar los poros y determinar su tamaño como, por ejemplo, las medidas de resonancia magnética nuclear (NMR, Nuclear Magnetic Resonance), difracción de rayos $\mathrm{X}$ tanto con ángulos pequeños como amplios, conductancia eléctrica, (26), etc. Glaves y Smith, (31), fueron los que propusieron la determinación del tamaño de poro en membranas saturadas con agua, usando la NMR. Las medidas de NMR primero se deben calibrar usando un material, el tamaño de cuyos poros se conozca, aunque es preciso asumir una geometría del poro. Las técnicas de difracción de rayos- $X$ de ángulo amplio permiten determinar el grado de cristalinidad del material, el cual puede estar relacionado con el tamaño de poro, (2); mientras que las de dispersión de rayos $X$ de ángulo pequeño proporcionan una información estructural importante de los poros a partir de las distintas heterogenei-

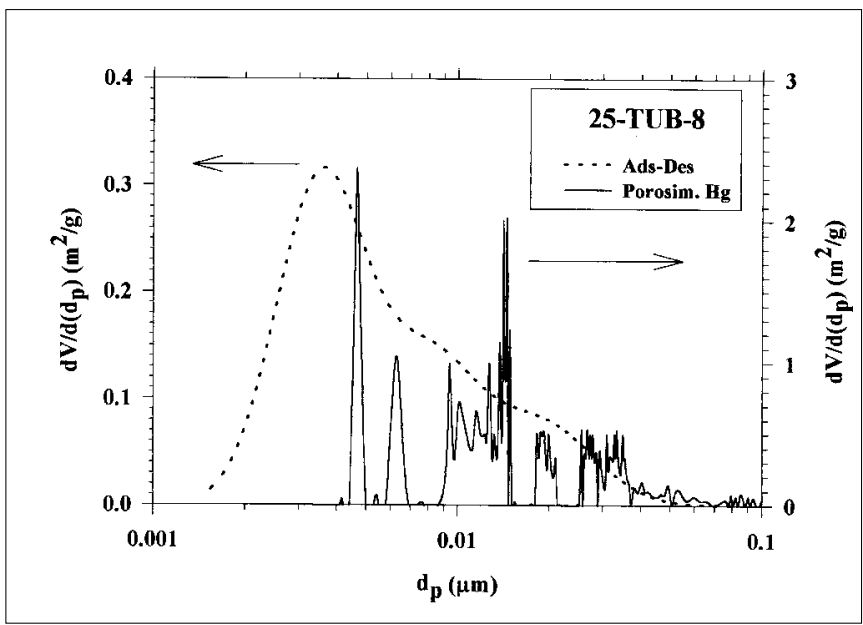

Fig. 11.- Se comparan las distribuciones obtenidas mediante porosimetría de mercurio y adsorción-desorción de nitrógeno para la membrana TAMI (25TUB8).

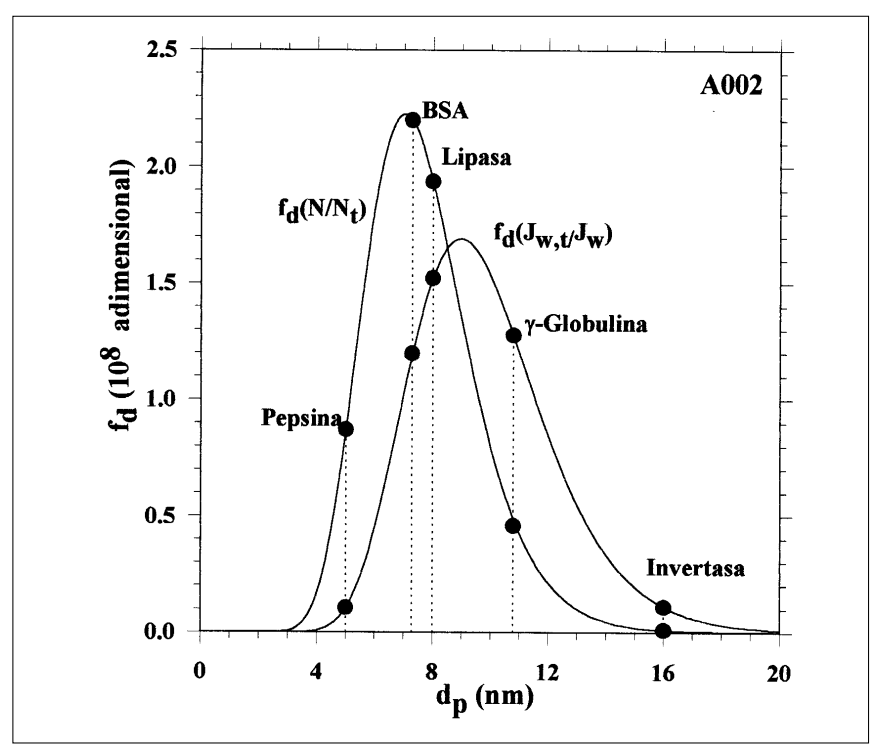

Fig. 12.- Distribución de tamaños de poro obtenida, para la membrana ANOPORE de $0,02 \mu \mathrm{m}$ de tamaño nominal de poro, mediante experimentos de retención de varias proteínas de tamaños moleculares crecientes. Se muestran las frecuencias tanto para número de poros como para los flujos, de modo que el área bajo las curvas es la unidad. 
dades de la densidad electrónica. Usando otras técnicas también se pueden obtener importantes parámetros físicos y químicos - como se hace en la espectroscopía electrónica para análisis químico (ESCA)- o bien analizar su composición química o la variación de ésta por adsorción de ciertas sustancias como se puede deducir de un análisis por espectroscopía de transformada de Fourier en el infrarrojo (FTIR) (32).

A la hora de elegir una determinada técnica, es interesante conocer cuales son sus principales características con el fin de elegir aquella que sea más interesante de acuerdo con el fin que se persigue y con la membrana que se esté utilizando. Un primer factor importante a la hora de elegir es el rango de tamaño de poros que cada técnica cubre (vease la Figura 4). Otro factor importante es la cantidad de muestra que se necesita para realizar la experiencia. Así, por ejemplo, mientras que en los métodos de punto de burbuja, retención de solutos o métodos microscópicos se necesita una pequeña cantidad de muestra, las técnicas de adsorción de gases o porosimetría de mercurio requieren una cantidad considerable de membrana, dependiendo de su porosidad. Hay que tener en cuenta también que hay ciertas técnicas, p.e. SEM o porosimetría de mercurio, que pueden destruir la muestra o como poco dañarla (especialmente la intrusión de mercurio añade además el riesgo de contaminación, dado el carácter nocivo de esta sustancia) (2).

El conocimiento de la estructura no sólo es importante para describir los efectos de filtrado, sino también, para estudiar las interacciones entre el material y el soluto; además, hay que tener presente que la mayor parte del área de las interfases está dentro de los poros. Por ello, muchas veces es necesario conocer también las propiedades eléctricas (potencial zeta, carga superficial, etc.) de la membrana ya que éstas actúan dentro de los poros afectando al transporte. Por todo ello conviene saber, en detalle, la geometría de los poros para así correlacionarla de forma adecuada con sus efectos en el flujo (2).

\section{APLICACIONES DE LOS PROCESOS DE MEMBRANA}

Existen multitud de aplicaciones en separación concentración y fraccionamiento en que los procesos de membrana tienen gran interés. Por destacar algunos, nos concentraremos en aquellos en que las membranas cerámicas han desplazado a las poliméricas de manera clara como resultado de sus características especiales. Estas aplicaciones se encuentran en varios grandes campos, (6): Separación de gases; reactores de membrana; industrias alimentaria y farmacéutica y Aguas y vertidos industriales.

Las membranas densas o de poro nano o subnanométrico son de gran utilidad en la separación de mezclas gaseosas. Se ha informado de separación de mezclas $\mathrm{CO}_{2} / \mathrm{CH}_{4}, \mathrm{H}_{2} / \mathrm{C}_{3} \mathrm{H}_{6}$, $\mathrm{N}_{2} / \mathrm{NH}_{3}, \mathrm{H}_{2} / \mathrm{O}_{2}, \mathrm{O}_{2} / \mathrm{NO}_{2}$ y $\mathrm{H}_{2} / \mathrm{N}_{2}$, entre otras, (6). En algunos casos parece existir una cierta actividad catalítica de la membrana permitiendo, por ejemplo un considerable enriquecimiento de mezclas 1:1:1 de $\mathrm{CH}_{4} / \mathrm{C}_{2} \mathrm{H}_{6} / \mathrm{C}_{3} \mathrm{H}_{8}$ aumentando hasta el 64 la masa de metano y decreciendo hasta casi eliminar el contenido de propano en una sola etapa.

En los reactores químicos se pueden emplear membranas cerámicas pasivas con el fin de separar productos de reactivos. Asimismo se pueden utilizar membranas con componentes catalíticos más o menos integrados en sus estructuras. La mayor parte de las reacciones químicas interesantes, en cuanto a la aplicación de membranas cerámicas, tienen lugar en fase gaseosa y a altas temperaturas. Incluyen: descomposición, deshidrogenización, hidrogenización, oxidación y desulfuriza- ción, entre otras.

En la industria alimentaria merecen la pena destacarse: las aplicaciones en la industria lechera, en el tratamiento de jugos de frutas, el procesado de bebidas alcohólicas o fermentadas. Mientras que son cada vez mas frecuentes las aplicaciones en Biotecnología, especialmente para fraccionamiento y separación de fermentos y productos deshechos en reactores enzimáticos, (33).

Finalmente, en la producción de agua potable, es muy utilizada la filtración en flujo tangencial sobre membranas cerámicas para eliminar materia orgánica así como partículas sólidas suspendidas. Por otra parte las membranas cerámicas son de gran aplicación en el tratamiento, para recuperación de compuestos valiosos o el ajuste de las propiedades de los vertidos con fines de conservación ecológica, de diversos vertidos de la: industria mecánica (lubricantes, refrigerantes, etc), la textil, la papelera y diversos ramos de la industria química (por ejemplo la industria del caucho).

\section{CONCLUSIONES}

Se ha mostrado de modo muy resumido la síntesis, caracterización y principales aplicaciones de las membranas cerámicas. Como resultado de sus características específicas, sobre todo, con altas resistencias química y térmica estas membranas son de gran interés en aplicaciones altamente exigentes en acidez, pH o temperatura de operación. Asimismo debido a la facilidad de limpieza y esterilización, su aplicación es muy adecuada en procesos de carácter altamente ensuciante. Algunos problemas de ingeniería y economía de fabricación impiden su uso todavía en aplicaciones menos exigentes.

\section{AGRADECIMIENTOS}

El trabajo realizado ha sido financiado con cargo a los siguientes proyectos de Investigación: QUI96-0767 del "Plan Nacional de Investigación y Desarrollo" (CICYT) y VA10496 de la Junta de Castilla y León.

\section{BIBLIOGRAFÍA}

1 M. Mulder, Basic Principles of Membrane Technology, Kluwer, Dordrecht, The Netherlands, 1991.

2 Hernández, A., Calvo, J.I., Prádanos, P., Palacio, L., "A multidisciplinary approach towards pore size distributions of microporous and mesoporous membranes", en Surface Chemistry and Electrochemistry, Smith-Sørensen, T. (Ed.), Marcel Dekker Inc., Nueva York, EEUU (1999).

3 R.E. Kesting, Synthetic Polymeric Membranes, A Structural Perspective, 2nd. ed., John Wiley and Sons, New York, NY, USA, 1985.

4 A. Hernández, P. Prádanos, J.I. Calvo, L. Palacio, "Metal Oxide Membranes" en Oxide surfaces, Ed. J. Wingrave, Marcel Dekker Inc., Nueva York, EEUU (En Prensa).

5 R.R. Bhave, Inorganic Membranes: Synthesis, Characteristics and Applications, Van Nostrand Reinhold, New York, USA, (1991).

6 K. Scott, Handbook of Industrial Membranes, Elsevier, Oxford, (1995).

7 J. Marchese, Membranas: Procesos con Membranas, Editorial Universitaria de San Luis, San Luis, Argentina, (1995).

8 S. Nakao, "Determination of pore size and pore size distribution. 3. Filtration membranes", J. Memb. Sci, 96 (1994) 131

9 L. Zeman, "Characterization of microfiltration membranes by image analysis of electron micrographs. Part. II. Functional and morphological parameters", J. Memb. Sci., 71 (1992) 233.

10 A. Hernández, J.I. Calvo, P. Prádanos, L. Palacio, M.L. Rodríguez and J.A. de Saja, "Surface structure of microporous membranes by computerised SEM image analysis applied to Anopore filters", J. Memb. Sci., 137 (1998) 89. 
11 G. Binnig, C.F. Quate and Ch. Gerber, "Atomic force microscopy", Phys. Rev. Lett., 56 (1986) 930.

12 A. K. Fritzsche, A.R. Arevalo, M.D. Moore, C.J. Weber, V.B. Elings, K. Kjoller and C.M. Wu, "The surface structure and morphology of polyvinylidene fluoride microfiltration membranes by atomic force microscopy", J. Memb. Sci, 68 (1992) 65.

13 P. Dietz, P.K. Hansma, O. Inacker, H.D. Lehmann and K.H. Herrmann, "Surface pore structures of micro- and ultrafiltration membranes imaged with the atomic force microscope", J. Memb. Sci., 65 (1992) 101.

14 Y. Kim and C.M. Lieber, "Machining oxide thin films with and atomic force microscope: pattern and object formation on the nanometer scale", Science, 257 (1992) 375.

15 H. Bechhold, M. Schlesinger, K. Silbereisen, L. Maier and W. Nurnberger, "Pore diameters of ultrafilters", Kolloid Z., 55 (1931) 172.

16 G. Reichelt, "Bubble point measurements on large areas of microporous membranes", J. Memb. Sci., 60 (1991) 253.

17 A. Hernández, J.I. Calvo, P. Prádanos and F. Tejerina, “Pore size distributions in microporous membranes. A critical analysis of the bubble point extended method", J. Memb. Sci., 112 (1996) 1.

18 ASTM F316, Standard Test Method for Pore Size Characteristics of Membrane Filters by Bubble Point and Mean Flow Pore Test.

19 ASTM E1294, Standard Test Methods for Pore Size Characteristics of Membrane Filters Using Automated Liquid Porosimeter.

20 S. Lowell and J.E. Shields, Powder Surface Area and Porosity, Powder Technology Series, B. Scarlett (Ser.Ed.), J. Wiley and Sons, New York, NY, USA, 1987.

21 E. Honold and E.L. Skau, "Applications of mercury intrusion method for determination of pore-size distribution of membrane filters", Science, 120 (1954) 805.

22 S.J. Gregg and K.S.W. Sing, Adsorption, Surface Area and Porosity, Academic Press, London, UK, 1982.

23 D. Dollimore and G.R. Heal, "An improved method for the calculation of pore size distribution from adsorption date", J. Appl. Chem., 14 (1964) 109.
24 K. Kaneko, "Determination of pore size and pore size distribution. 1. Adsorbents and catalysis", J. Memb. Sci., 96 (1994) 59.

25 L. Palacio, P. Prádanos, J.I. Calvo and A. Hernández, "Porosity determinations by a new gas penetration volumetric method", Acta Polytech. Scandinavica, 247 (1997) 115.

26 K. Sakai, "Determination of pore size and pore size distribution. 2. Dialysis membranes", J. Memb. Sci., 96 (1994) 91.

27 M. Brun, A. Lallemand, J.F. Quinson and C. Eyraud, “Changement d'état liquide - solide dans les milieux poreux. I. Étude experimentale de la solidification de l'eau et du benzène", J. Chim. Phys., 70 (1973) 973.

$28 \mathrm{M}$. Sarbolouki, "A general diagram for estimating pore size of ultrafiltration and reverse osmosis membranes", Sep. Sci. and Technol., 17 (1982) 381.

29 R. Nobrega, H. de Balmann, P. Aimar and V. Sánchez, “Transfer of dextran through ultrafiltration membranes: a study of rejection data analysed by gel permeation chromatography", J. Memb. Sci., 45 (1989) 17.

30 V. Gekas, G. Trägårdh and B. Hallström, Ultrafiltration Membrane Performance Fundamentals, Swedish Foundation for Membrane Technology, Lund, Sweden, (1993).

31 C.L. Glaves and D.M. Smith, "Membrane pore structure analysis via NMR spin lattice relaxation experiments", J. Memb. Sci., 46 (1989) 167.

32 A. Pihlajamäki, P. Väisänen and M. Nyström, "Characterization of clean and fouled polymeric ultrafiltration membranes by Fourier transform IR spectroscopy attenuated total reflection", Colloid \& Surfaces A, 138 (1998) 323.

33 G. Cueille and M. Ferreira, "place of mineral membranes in the processes for bio-industry and the food-industry", Proc. $1^{\text {st }}$ Intl. Conf. Inorganic Membranes, 3-6 July, (1990), Montpellier, France.

Recibido: $20-10-98$

Aceptado: 30-3-99 